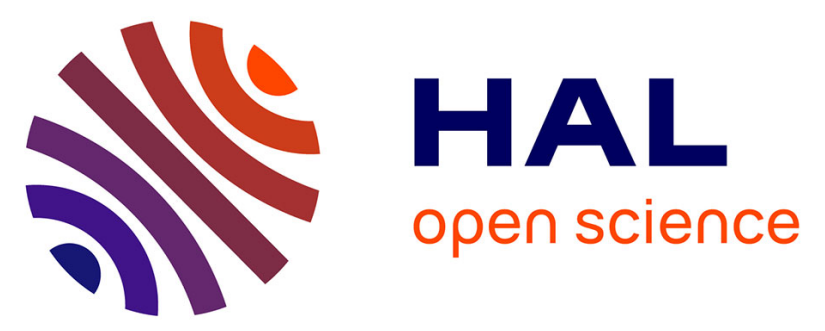

\title{
On the influence of electron mobilities on the yields of formation of positronium in liquids: New I3 measurements and Monte Carlo simulations
}

Isabelle Billard, Thomas Goulet, Jean-Paul Jay-Gerin, André Bonnenfant

\section{- To cite this version:}

Isabelle Billard, Thomas Goulet, Jean-Paul Jay-Gerin, André Bonnenfant. On the influence of electron mobilities on the yields of formation of positronium in liquids: New I3 measurements and Monte Carlo simulations. Journal of Chemical Physics, 1998, 108 (6), pp.2408-2415. 10.1063/1.475624 . hal-02272002

\section{HAL Id: hal-02272002 \\ https://hal.science/hal-02272002}

Submitted on 27 Aug 2019

HAL is a multi-disciplinary open access archive for the deposit and dissemination of scientific research documents, whether they are published or not. The documents may come from teaching and research institutions in France or abroad, or from public or private research centers.
L'archive ouverte pluridisciplinaire HAL, est destinée au dépôt et à la diffusion de documents scientifiques de niveau recherche, publiés ou non, émanant des établissements d'enseignement et de recherche français ou étrangers, des laboratoires publics ou privés. 


\title{
On the influence of electron mobilities on the yields of formation of positronium in liquids: New $I_{3}$ measurements and Monte Carlo simulations
}

\author{
Isabelle Billard ${ }^{\mathrm{a})}$ \\ Institut de Recherches Subatomiques, Chimie Nucléaire et Physico-Chimie des Rayonnements, \\ CNRS/IN2P3-ULP, Bâtiment 35, B.P. 28, 67037 Strasbourg Cedex 2, France \\ Thomas Goulet and Jean-Paul Jay-Gerin \\ Département de Médecine Nucléaire et de Radiobiologie, Faculté de Médecine, Université de Sherbrooke, \\ Sherbrooke (Québec) J1H 5N4, Canada \\ André Bonnenfant \\ Institut de Recherches Subatomiques, Chimie Nucléaire et Physico-Chimie des Rayonnements, \\ CNRS/IN2P3-ULP, Bâtiment 35, B.P. 28, 67037 Strasbourg Cedex 2, France
}

(Received 26 June 1997; accepted 29 October 1997)

\begin{abstract}
We study the formation of positronium (Ps) at the end of the radiation tracks generated by the passage of energetic positrons in various liquids. We focus particularly on the possible influence of the thermal electron mobility $\mu\left(e^{-}\right)$on this process. To this end, we present a compilation of experimental data of the probability of ortho-Ps formation $I_{3}$ and $\mu\left(e^{-}\right)$in 51 pure liquids at room temperature. We also present new measurements of $I_{3}$ as a function of temperature for $n$-hexane, 2,2-dimethylbutane, tetramethylsilane, and tetramethylstannane from the melting point up to $294 \mathrm{~K}$. Those results are compared to the variation of $\mu\left(e^{-}\right)$with temperature which can be found in the literature. Finally, we use Monte Carlo simulation techniques to perform a sensitivity study in which we evaluate the relative contributions of various physicochemical properties of the liquids (such as the mean thermalization distances of $e^{+}$and $e^{-}$, their thermal mobilities, the dielectric constant, and the temperature) on the values of $I_{3}$. A review of previous works on this question is also given.

(C) 1998 American Institute of Physics. [S0021-9606(98)50206-1]
\end{abstract}

\section{INTRODUCTION}

The positron $\left(e^{+}\right)$, the antiparticle of the electron $\left(e^{-}\right)$, when injected with a certain amount of kinetic energy in a liquid, appears to be an ionizing particle in many ways comparable to an electron of the same energy. Indeed, both have the same mass and the same absolute charge. However, positrons present some specific characteristics making a direct transposition of models and experimental results from one particle to another difficult. The opposite electric charge of $e^{+}$and $e^{-}$leads, for example, to differences in the potentials experienced by those particles. Moreover, it is clear that, contrary to an electron, a positron cannot recombine with one of the molecules that it has ionized within its track.

It is well known that the ultimate fate of a positron in matter is its annihilation with an $e^{-}$of the medium. There is a possibility, however, that the encounter pair $\left(e^{+}, e^{-}\right)$forms a transient bound state called "positronium" (Ps). In this way, the formation of Ps can be regarded as a peculiar recombination process. According to a standard convention, the probability of Ps formation consists of two terms, $I_{1}$ and $I_{3}$, which correspond to the formation of para-positronium and ortho-positronium, respectively. The remaining probability $I_{2}=1-\left(I_{1}+I_{3}\right)$ is associated to the case where the $e^{+}$

\footnotetext{
a) Author to whom correspondence should be addressed. Electronic mail: fastrade@in2p3.fr
}

does not form Ps but annihilates directly with an electron. The spur model ${ }^{1-3}$ describing Ps formation is based on the knowledge we have of the primary processes occurring in a liquid, as deduced from radiation chemistry results. It also includes the specific properties of $e^{+}$and Ps. This model essentially states that the electrons involved in the formation of Ps are not those that are bound to molecules, but the quasifree ones that originate from the numerous ionization events of the radiation track. The name "spur" refers to the fact that those electrons tend to belong to more or less isolated clusters associated to neighboring ionization events. On that basis, positrons can hardly be studied on their own because their ionizing properties, as stressed above, lead to the concomitant presence of a large number of electrons. The respective effects of each of these particles are therefore intricately entangled.

Nevertheless, one may take advantage of the specific properties of $e^{+}$to improve our knowledge of the electron spur and more particularly of the radiation track ends where the Ps are formed. When looking for a comprehensive model of primary processes in a liquid, the results derived from positron annihilation techniques should be taken into account.

In this paper, we try to join radiation chemistry interests and positron annihilation data to highlight the possible mutual benefits each of these communities could gain from more work done conjunctively. We decided to center this 
study on the possible influence of the thermal electron mobility $\mu\left(e^{-}\right)$on Ps formation. First, we gather a wide compilation of $I_{3}$ data in a number of pure liquids of known $\mu\left(e^{-}\right)$. Second, we present new experimental results on the variation of $I_{3}$ with temperature (between the melting point and $294 \mathrm{~K}$ ) in four liquids, namely, tetramethylsilane (TMS), 2,2-dimethylbutane (2,2-DMB), $n$-hexane, and tetramethylstannane (TMSn). In the first three of those liquids, the possible correlation between $I_{3}$ and $\mu\left(e^{-}\right)$can be examined since measurements of the temperature dependence of $\mu\left(e^{-}\right)$exist in the literature. For TMSn, this information is not available and we submit our data as an open challenge for further measurements. Finally, we describe the process of Ps formation with Monte Carlo simulation techniques to get some insight on the conditions in which it can occur. In particular, we investigate the respective roles played by different variables of the problem, such as the mean thermalization distances of $e^{-}$and $e^{+}$, the thermal mobilities of those particles, and the dielectric constant of the considered medium.

\section{EXPERIMENT}

Tetramethylsilane, tetramethylstannane, 2,2-dimethylbutane, and $n$-hexane, reagent grade from Aldrich, were used as received, without further purification. For each sample, an ampule containing approximately $4 \mathrm{~cm}^{3}$ of liquid and a $5.55 \mathrm{~Bq}^{22} \mathrm{Na}$ source $\left(\beta^{+}\right.$emitter) deposited on a thin glass foil was prepared by due degassing as previously described. ${ }^{4}$ The percentage of positrons emitted by the ${ }^{22} \mathrm{Na}$ and annihilating in the glass foil instead of in the liquid was $8 \%$. The glass container was sealed under vacuum to avoid oxygen contamination. The cooling system is composed of a liquid-nitrogen container over which the cryostat is fixed. The temperature adjustment is achieved through gaseous exchange and the whole cooling and temperature control process is monitored by a dedicated computer. The temperature was controlled within $0.1 \mathrm{~K}$ over all the investigated range.

The lifetime spectroscopy (LS) apparatus is a classical setup, composed of two photomultipliers and scintillators, connected to a basic electronic device, as extensively described elsewhere. ${ }^{5}$ The emission of a positron in the liquid is monitored via the detection of the $1.28 \mathrm{MeV}$ photon which readily follows the $\beta^{+}$decay of the ${ }^{22} \mathrm{Na}$ atom, using a traditional Pilot U scintillator and a XP2020 photomultiplier (Philips). The instant of positron annihilation is determined through the detection of one of the two photons emitted at that time. In this case, a $\mathrm{BaF}_{2}$ scintillator and a XP2020Q photomultiplier (quartz window) have been used to take advantage of the high-energy selectivity of such a scintillator. The low activity of the source ensures the presence of only one positron at a time in the sample. The temporal resolution of the LS setup was $230 \mathrm{ps}$, as measured as the full width at half maximum of the ${ }^{60} \mathrm{Co}$ prompt curve.

The LS spectra were decomposed in a sum of three exponential decays, of relative intensities $I_{i}$ (in \%) and characteristic decay times $\tau_{i}$, convoluted by the measured apparatus response. These components are ascribed, with increasing subscript $i$, to the three considered fates of the positron in a pure solvent: the singlet para-positronium ( $p-\mathrm{Ps})$, the $e^{+}$ remaining free (that is, not having formed Ps), and the triplet ortho-positronium ( $o$-Ps, with substates $m=-1,0,+1)$. Owing to the rather low value of $\tau_{1}(\cong 125 \mathrm{ps})$, the lifetime of singlet Ps, no reliable value of both $\tau_{1}$ and $I_{1}$ can be deduced from a LS fit because of inherent correlation of the two parameters that describe the shortest-lived component. Due to the statistical population of the substates of positronium in the temperature range investigated, the ratio $I_{3} / I_{1}$ should be strictly equal to 3 . One should note that, in some cases, a slight deviation to this ratio has been evidenced and attributed to the small influence of the medium on the wave function of Ps. ${ }^{6,7}$ However, previous experiments ${ }^{8}$ showed that, in the case of $n$-hexane, this effect gives a ratio of 3.12 and is independent of temperature over a large range. As a consequence, we will consider throughout this study that the value of $I_{3}$ corresponds to $75 \%$ of the total amount of Ps formed. The LS data will be presented in terms of $I_{3}, \tau_{3}$, and $\tau_{2}$, obtained in a multiparametric fit in which none of the parameters $I_{i}$ and $\tau_{i}$ were arbitrarily fixed.

\section{RESULTS AND DISCUSSION}

\section{A. Compilation of data}

Among the physical properties of the liquids that may influence the formation of Ps, Jansen and Mogensen ${ }^{9}$ have suggested that the thermal electron mobility $\mu\left(e^{-}\right)$could be a determinant one. This assumption relied on a comparison of 12 solvents, for which these authors reported a "strong correlation" between $I_{3}$ and $\mu\left(e^{-}\right)$. A priori, the mobility of the electron has to play a role in Ps formation because of the necessity of a $e^{+}-e^{-}$encounter. However, this correlation might be more intricate than originally envisioned. In this paper, we extend the comparison to a wider range of liquids and point out the difficulties in reaching a clear conclusion on this subject.

Table I presents a new compilation of $I_{3}$ and $\mu\left(e^{-}\right)$ obtained at room temperature for 51 pure liquids. ${ }^{9-30}$ The same data can be visualized in Fig. 1, along with the values reported by Jansen and Mogensen. ${ }^{9}$ A first glance at Fig. 1 seems to confirm the previously reported correlation. However, if one makes a distinction between low-, intermediate-, and high-mobility solvents, the existence of a universal correlation between $I_{3}$ and $\mu\left(e^{-}\right)$becomes questionable. For $e^{-}$mobilities below $\sim 4 \times 10^{-3} \mathrm{~cm}^{2} \mathrm{~V}^{-1} \mathrm{~s}^{-1}, I_{3}$ is approximately constant and equal to $\sim 23 \%$. For $\mu\left(e^{-}\right)$between $4 \times 10^{-3}$ and $1 \mathrm{~cm}^{2} \mathrm{~V}^{-1} \mathrm{~s}^{-1}$, the values of $I_{3}$ are larger $(\sim 40 \%)$ but remain rather independent of $\mu\left(e^{-}\right)$. A continuous positive correlation between $I_{3}$ and $\mu\left(e^{-}\right)$appears only above $1 \mathrm{~cm}^{2} \mathrm{~V}^{-1} \mathrm{~s}^{-1}$, that is, for high-mobility solvents. This last observation echoes the well-known relation between the free-ion yields and $\mu\left(e^{-}\right)$in high-mobility solvents. ${ }^{10}$

One should note that the absence of correlation in the case of polar solvents can be understood in the framework of the spur model describing Ps formation. In fact, following this model, positronium is formed either with quasifree or with weakly trapped electrons, ${ }^{27}$ but not with fully solvated electrons. ${ }^{31,32}$ Therefore, there is no reason to observe any correlation between the solvated electron mobility (the only 
TABLE I. $o$-Ps formation probability $I_{3}$ and thermal electron mobility $\mu\left(e^{-}\right)$in a variety of pure liquids. ${ }^{\mathrm{a}}$

\begin{tabular}{|c|c|c|c|}
\hline Liquid & $\begin{array}{l}I_{3} \\
(\%)\end{array}$ & $\begin{array}{c}\mu\left(e^{-}\right) \\
\left(\mathrm{cm}^{2} \mathrm{~V}^{-1} \mathrm{~s}^{-1}\right)\end{array}$ & References \\
\hline Tetramethylsilane & $59.0^{\mathrm{b}}$ & 102 & 10,11 \\
\hline Tetramethylstannane & $56.5^{\mathrm{b}}$ & 75 & 10,11 \\
\hline $\begin{array}{l}\text { 2,2-dimethylpropane } \\
\text { (neopentane) }\end{array}$ & 52.5 & 69.5 & 10,12 \\
\hline $\begin{array}{l}\text { 2,2-dimethylbutane } \\
\text { (neohexane) }\end{array}$ & $46.1^{\mathrm{b}}$ & 12 & 10 \\
\hline $\begin{array}{l}\text { 2,2,4-trimethylpentane } \\
\text { (isooctane) }\end{array}$ & 42.4 & 6.5 & $10,12-14$ \\
\hline 2,3-dimethylbutene-2 & 43.7 & 6 & 10,15 \\
\hline cis-butene-2 & 49 & 2.2 & 9,10 \\
\hline 2-methylpentane & 36.5 & 0.29 & 10,15 \\
\hline Cyclohexane & 38.1 & 0.28 & $10,12,16$ \\
\hline 3-methylpentane & 37.1 & 0.2 & 10,15 \\
\hline $\begin{array}{l}\text { 1,3,5-trimethylbenzene } \\
\text { (mesitylene) }\end{array}$ & 46 & 0.17 & 10,12 \\
\hline$n$-pentane & 41 & 0.154 & 10,12 \\
\hline Benzene & 43.5 & 0.125 & $10,12-14,17$ \\
\hline cis-decalin & 33.6 & 0.1 & 10,18 \\
\hline $\begin{array}{l}\text { Methylbenzene } \\
\text { (toluene) }\end{array}$ & 42.6 & 0.075 & $10,12,13$ \\
\hline$n$-hexane & $41.8^{\mathrm{b}}$ & 0.074 & 10 \\
\hline $\begin{array}{l}\text { 1,3-dimethylbenzene } \\
\text { (m-xylene) }\end{array}$ & 45 & 0.071 & $10,12,19$ \\
\hline $\begin{array}{l}\text { 1,4-dimethylbenzene } \\
\text { (p-xylene) }\end{array}$ & 45 & 0.066 & 10,12 \\
\hline Methylcyclohexane & 41 & 0.057 & 10,12 \\
\hline$n$-heptane & 41 & 0.051 & 10,20 \\
\hline trans-butene-2 & 45 & 0.029 & 9,10 \\
\hline$n$-decane & 39.6 & 0.025 & 10,12 \\
\hline $\begin{array}{l}\text { 1,2-dimethylbenzene } \\
\text { (o-xylene) }\end{array}$ & 44 & 0.021 & $10,12,19$ \\
\hline 1,2,3,4-tetramethylbenzene & 46 & 0.02 & 10,12 \\
\hline$n$-dodecane & 38 & 0.02 & 10,12 \\
\hline$n$-tetradecane & 36 & 0.016 & 10,12 \\
\hline trans-decalin & 37.3 & 0.013 & 10,16 \\
\hline Hexafluorobenzene & 51 & 0.011 & 10,12 \\
\hline Diethyl ether & 32 & 0.0086 & 12,21 \\
\hline Carbon disulfide & 44 & 0.0078 & $12,13,22$ \\
\hline Tetrahydrofuran (THF) & 29.3 & 0.0067 & 21,23 \\
\hline Triethylamine & 42.9 & $0.0045^{\mathrm{c}}$ & 23,25 \\
\hline$n$-butylamine & 29.9 & 0.0027 & 15,21 \\
\hline$N, N$-dimethylformamide & 18.2 & $0.0023^{\mathrm{c}}$ & 26 \\
\hline $\begin{array}{l}\text { Hexamethylphosphoric } \\
\text { triamide (HMPT or HMPA) }\end{array}$ & 27.5 & 0.0021 & 21,27 \\
\hline Tributylamine & 35.3 & 0.002 & 15,21 \\
\hline$N, N$-dimethylacetamide & 22.6 & $0.002^{\mathrm{c}}$ & 28 \\
\hline Water $\left(\mathrm{H}_{2} \mathrm{O}\right)$ & 28 & 0.00191 & 12,21 \\
\hline Deuterium oxide $\left(\mathrm{D}_{2} \mathrm{O}\right)$ & 23 & 0.00151 & 12,21 \\
\hline $\begin{array}{l}\text { Dimethylsulfoxide } \\
\text { (DMSO) }\end{array}$ & 14.3 & $0.0011^{\mathrm{c}}$ & 20,27 \\
\hline 1,4-dioxane & 53.5 & 0.0009 & $12,14,21$ \\
\hline 1-butanol & 23.4 & 0.00075 & 12,21 \\
\hline Methanol & 22 & 0.00062 & 21,29 \\
\hline 2-propanol & 22.1 & 0.00051 & 15,21 \\
\hline 1-propanol & 22.9 & 0.00046 & 12,21 \\
\hline Ethanol & 22 & 0.00037 & 12,21 \\
\hline 1-octanol & 24.5 & $0.00036^{\mathrm{c}}$ & 12 \\
\hline $\begin{array}{l}\text { 1,2-ethanediol } \\
\text { (ethylene glycol) }\end{array}$ & 22.3 & 0.00028 & 21,29 \\
\hline 1,3-propanediol & 22.2 & $0.000083^{\mathrm{c}}$ & 29 \\
\hline Cyclohexanol & 26 & $0.000063^{c}$ & 9,21 \\
\hline $\begin{array}{l}\text { 1,2,3-propanetriol } \\
\text { (glycerol) }\end{array}$ & 24 & 0.0000075 & 21,30 \\
\hline
\end{tabular}

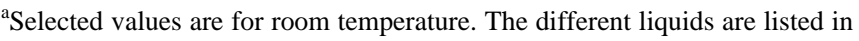
the order of decreasing $\mu\left(e^{-}\right)$.

bresent work.

${ }^{c}$ Estimated from the empirical relation $\mu\left(e^{-}\right)\left(\right.$in $\left.\mathrm{cm}^{2} \mathrm{~V}^{-1} \mathrm{~s}^{-1}\right)=C / \eta^{p}$, where $\eta$ (in centipoise) is the viscosity of the solvent, $C=1.92 \times 10^{-3}$, and $p=0.84$ (see Ref. 24).

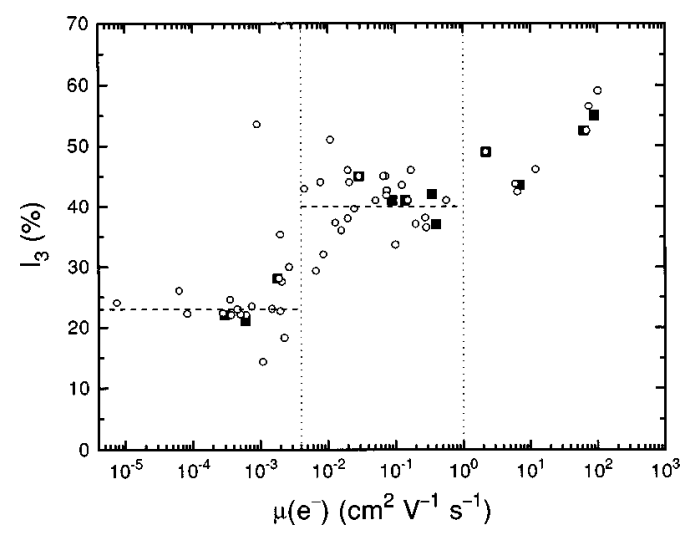

FIG. 1. Variation of the $o$-Ps formation probability $I_{3}$ with the thermal electron mobility $\mu\left(e^{-}\right)$(in $\mathrm{cm}^{2} \mathrm{~V}^{-1} \mathrm{~s}^{-1}$ ) in a variety of liquids at ambient temperature. $\bigcirc$ : values given in Table I; $\mathbf{\square}$ : values reported in Ref. 9 and on which the idea of a $I_{3}$ vs $\mu\left(e^{-}\right)$correlation was originally based. The vertical dotted lines define three ranges of $\mu\left(e^{-}\right)$in which $I_{3}$ has specific behaviors (see text). Those divisions, which are based on a visual inspection of the present compilation, are essentially phenomenological. One can note that the intermediate group $\left[4 \times 10^{-3}<\mu\left(e^{-}\right)<1 \mathrm{~cm}^{2} \mathrm{~V}^{-1} \mathrm{~s}^{-1}\right]$, which consists mainly of nonpolar liquids, also includes some weakly polar solvents that display similar properties as far as Ps formation is concerned.

one to be measured in polar liquids) and the Ps formation yield. As for the absence of correlation between $I_{3}$ and $\mu\left(e^{-}\right)$among the low-mobility nonpolar solvents, it indicates that the factors determining the yields of Ps formation are not necessarily the same for all the considered liquids. It is important to realize here that in comparing the values of $I_{3}$ for different liquids at a given temperature, $\mu\left(e^{-}\right)$is not the only parameter which is varied. Various other physicochemical properties which could influence the formation of Ps are specific for each liquid. One can think, for example, of the dielectric constant of the medium, the related Onsager radius, the solvent density, and the mean thermalization distances of $e^{-}$and $e^{+}$.

In order to overcome this intrinsic limitation of solventto-solvent comparisons, Mogensen and co-workers ${ }^{33}$ also studied the influence of temperature $(T)$ on $I_{3}$ in a single solvent, neopentane, to assess more firmly the bases of the foreseen correlation. From their study, ranging from the melting point of neopentane up to its critical temperature, the idea of a $I_{3}$ vs $\mu\left(e^{-}\right)$correlation was reinforced by the fact that the variation with temperature of those two quantities displayed some similarities. However, one should keep in mind that, even in a given liquid, $\mu\left(e^{-}\right)$is not the only physical property that varies with $T$. The lack of theoretical background stopped any further attempts of these authors to handle the problem.

At this stage, two remarks are in order:

(i) The dependence of $I_{3}$ on the physicochemical parameters of a given liquid is obviously a multiparametric problem, and temperature does not appear to be a "good" parameter, because all other quantities vary along with it. Some variations are small, such as that of the dielectric constant $\epsilon$, and some are important, such as that of the density. Therefore, in such a case, the Ps formation yield in a given liquid might not appear to be dependent on $\epsilon$, whereas density would seem to be strongly correlated to $I_{3}$. Under these 


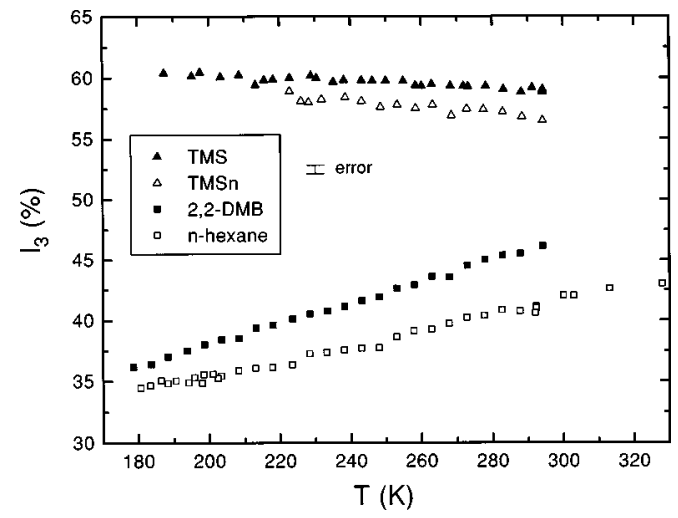

FIG. 2. Variation with temperature of the $o$-Ps formation probability $I_{3}$ in tetramethylsilane (TMS), tetramethylstannane (TMSn), 2,2-dimethylbutane (2,2-DMB), and $n$-hexane.

conditions, the joint variation of two parameters with $T$ must be examined with caution before concluding to the existence of any physical correlation. As an example of correlations to be examined with caution, note the work of Gee and Freeman, ${ }^{22}$ who also mentioned the $I_{3}$ vs $\mu\left(e^{-}\right)$correlation first stated by Mogensen, and added the correlation of $\tau_{3}$ with $\mu\left(e^{-}\right)$. They pointed out that this observation was offered "without interpretation." In fact, the annihilation process of $o$-Ps whose characteristic time is $\tau_{3}$, is a nuclear phenomena occurring on the nanosecond time scale and sensitive mainly to the electronic density of the medium. This annihilation predominantly occurs with one of the outer electrons of the molecules surrounding Ps (the so-called pick-off process) and there are no basic physical reasons that could explain a direct causal relation between $\mu\left(e^{-}\right)$and $\tau_{3}$.

(ii) In the specific case of neopentane, ${ }^{33}$ it can be noted that both $I_{3}$ and $\mu\left(e^{-}\right)$level off a few tens of degrees before the critical point, but the ranges of $T$ values in which these quantities remain constant do not coincide exactly. In our opinion, this is evidence that $I_{3}$ does not depend solely on the value of $\mu\left(e^{-}\right)$. Thus in order to get an improvement of our knowledge of the intricate process of Ps formation, one has to handle the whole problem together.

\section{B. New data}

Taking into account the previous remark about the multiparametric aspect of the problem, we chose to focus on three liquids (TMS, 2,2-DMB, and $n$-hexane) for which the literature provided a good deal of information on the temperature dependence of density, electron thermalization distance, and $\mu\left(e^{-}\right)$. Figures 2-4 show the variation with $T$ of $I_{3}, \tau_{2}$, and $\tau_{3}$, respectively, in these three liquids and in TMSn, a solvent for which much less information is available. In the case of $n$-hexane, the values of $I_{3}$ above $294 \mathrm{~K}$ shown in Fig. 2 are taken from a previous study, ${ }^{8}$ where $I_{3}$ was measured from $264 \mathrm{~K}$ up to $338 \mathrm{~K}$. The two sets of measurements are in agreement in the overlapping temperature range. As can be seen from Fig. 2, the four liquids studied could be separated into two distinct groups. On the one hand, the $I_{3}$ values in TMS and TMSn are large and decrease slightly with $T$. On the other hand, the $I_{3}$ values in

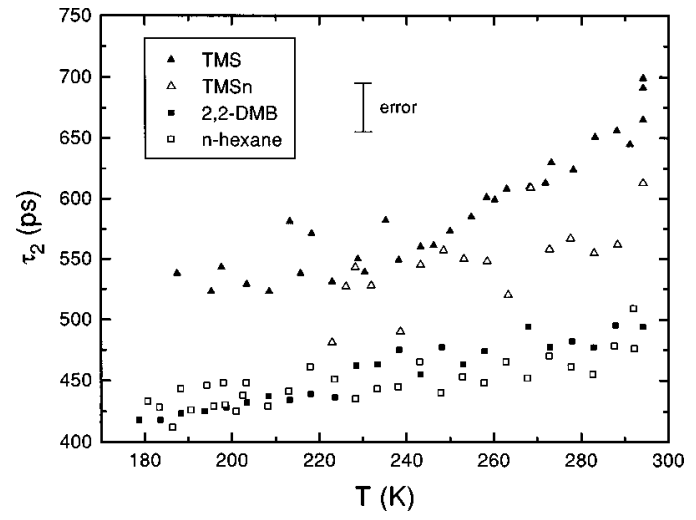

FIG. 3. Variation with temperature of the lifetime $\tau_{2}$ (in ps) of the free positron in tetramethylsilane (TMS), tetramethylstannane (TMSn), 2,2dimethylbutane (2,2-DMB), and $n$-hexane.

2,2-DMB and $n$-hexane are smaller and increase with $T$. One can note that the temperature dependence is more pronounced in the second group than in the first one. This distinction between two groups is less clear, but still visible, in the $\tau_{2}$ values, as illustrated in Fig. 3. In TMS and TMSn, our values of $\tau_{2}$ are rather high, as compared to those found in most solvents, but are in agreement with another determination for TMS. ${ }^{34}$ In contrast to what was seen in Figs. 2 and 3 concerning a possible classification of the four solvents into two groups, Fig. 4 displays no such grouping of the data. For instance, TMS and TMSn, presenting similar high $I_{3}$ and $\tau_{2}$ values, are the most different of our four liquids in terms of their $\tau_{3}$ values. In all cases, we find that $\tau_{3}$ increases monotonically with $T$, a phenomenon usually observed, which is attributed to a decrease in density. ${ }^{28}$

When comparing the variation with $T$ of $I_{3}$ in $n$-hexane and in 2,2-DMB to that of $\mu\left(e^{-}\right)$in the same liquids, ${ }^{35,36}$ we find that both $I_{3}$ and $\mu\left(e^{-}\right)$display a rather steady increase. ${ }^{37}$ This similarity of temperature dependence of those two quantities supports the observation that was made by Jacobsen et al. $^{33}$ in neopentane. In TMS, however, the situation is quite different. In fact, as can be seen in Fig. 5, $\mu\left(e^{-}\right)$passes through a minimum around $260 \mathrm{~K}$ in this highmobility liquid, ${ }^{38}$ whereas $I_{3}$ appears to decrease monotonically in the temperature range investigated. This result sug-

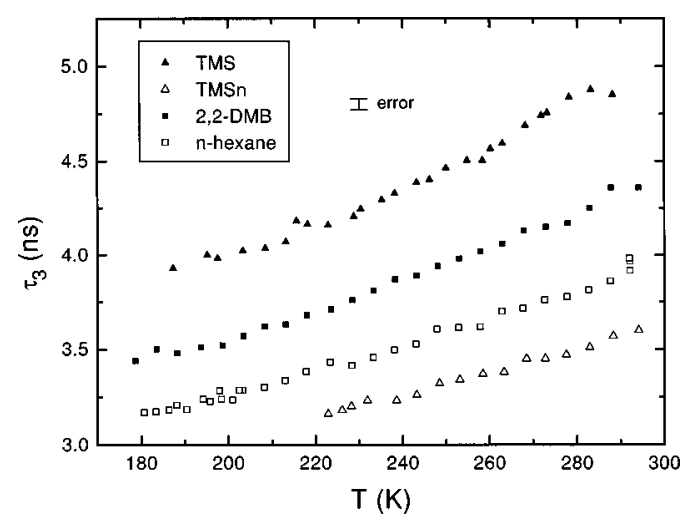

FIG. 4. Variation with temperature of the lifetime $\tau_{3}$ (in ns) of the $o$-Ps in tetramethylsilane (TMS), tetramethylstannane (TMSn), 2,2-dimethylbutane (2,2-DMB), and $n$-hexane. 


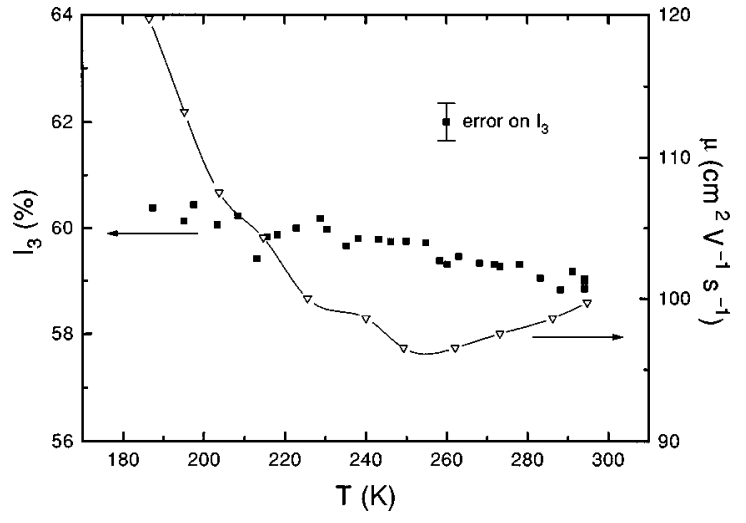

FIG. 5. Variation with temperature of the $o$-Ps formation probability $I_{3}$ and of the thermal electron mobility $\mu\left(e^{-}\right)$(Ref. 38) in tetramethylsilane.

gests that $I_{3}$ does not depend solely on the value of $\mu\left(e^{-}\right)$. In view of the fact that the variations of $\mu\left(e^{-}\right)$and $I_{3}$ involved in this comparison are rather small, it would be useful to extend the measurements of $I_{3}$ to higher temperatures in order to further substantiate this conclusion.

\section{Monte Carlo simulations}

\section{General approach}

In order to interpret the observed temperature dependence of $I_{3}$ and its variation from one solvent to another, we use Monte Carlo simulation techniques to describe the fate of quasifree positrons in the tracks that they generate in a liquid. The reason for resorting to a numerical approach rather than to an analytical one lies mainly in the complexity of the problem at hand. In fact, as was explained above, a number of factors influence the probability that the $e^{+}$encounters one of the quasifree $e^{-}$that it creates by ionizing the surrounding medium. Moreover, many of those factors cannot be quantified from direct measurements or from first principles, especially those related to the transport properties of $e^{+}$. We are thus led to conduct a sensitivity study that consists of assessing the role played by various physical quantities in the observed temperature dependence of $I_{3}$.

The probability of Ps formation is essentially determined by the competition between three processes: the encounter of $e^{+}$and $e^{-}$, the recombination of the geminate pair $\left(e^{-}, \mathrm{S}^{+}\right)$, and the first-order decay of $e^{+}$due to its annihilation with a bound electron of the medium. Since Ps formation is thought to occur at the end of the primary $e^{+}$track, it would be quite unrealistic to disregard the relative proximity of the energy deposition events in this region. ${ }^{39}$ As was done in a similar Monte Carlo simulation study of Ps formation, ${ }^{40}$ we found it important to include more than one $\left(e^{-}, \mathrm{S}^{+}\right)$pair in our modelized spur. This multi-ionization nature of the spur is determined by a comparison of the most probable thermalization distances $b_{e^{-}}$and $b_{e^{+}}$to the average distance $D$ between neighboring ionization sites. The parameters of the model are thus: (i) the thermalization distance distribution of both $e^{+}$ and $e^{-}$, (ii) the average distance $D$ between successive ionizations in the track end, (iii) the thermal mobility $\mu\left(e^{+}\right)$ and $\mu\left(e^{-}\right)$of those two particles (the mobility of the cation $\mathrm{S}^{+}$is generally negligible), (iv) the dielectric constant which affects the strength of the Coulombic interactions in the medium, and $(\mathrm{v})$ the characteristic decay time $\tau_{2}$ of the $e^{+}$.

\section{Simulations}

The first stage of a simulation run consists of generating a set of initial positions for a single thermalized $e^{+}$as well as for the cations $\mathrm{S}^{+}$and the thermalized $e^{-}$that lie in the $e^{+}$ track end. The thermalization of $e^{-}$and $e^{+}$does not have to be explicitly simulated here since $b_{e^{-}}$and $b_{e^{+}}$can be stochastically sampled from the Gaussian distributions that were obtained for $e^{-}$from previous free-ion-yield studies. ${ }^{41,42}$ One should note that the trajectories of the thermalizing $e^{+}$and $e^{-}$are essentially independent of each other because the average kinetic energy of those "hot' particles $(\sim 2 \mathrm{eV})$ is much larger than the potential energy associated to their mutual interaction. Moreover, since many scatterings are involved in the thermalization process, the geometry of the positions of the species is independent of the angle at which the electron is ejected during the ionization of $S$ by $e^{+}$. For simplicity, we fix the geometry of the track end as consisting of a linear string of $N_{i}$ equidistant ionization sites where the cations $\mathrm{S}^{+}$remain immobile and from which the electrons start the random walk that leads to their progressive thermalization. In the model considered here, the thermalization process for the $e^{+}$is assumed to start from the last one of those ionization sites.

In the second stage of the simulation, the Brownian motion and encounters of those thermalized species is followed with a step-by-step procedure ${ }^{43,44}$ until the fate of the $e^{+}$is determined. The simulation of the motion of the particles is performed with a Monte Carlo code that decomposes the trajectory of the $N_{i}$ electrons and of the $e^{+}$into a series of stochastically sampled relaxation times during which the "free paths" of the particles are determined classically, accounting for the net Coulomb field experienced by the charges. To take into account the dynamic nature of the electric field (since the whole set of charges is continually moving), the relaxation times are subdivided into smaller time intervals of the order of $10^{-15} \mathrm{~s}$. The distribution of relaxation times is exponential and its average value is directly proportional to the mobility of the particle. ${ }^{44}$ The free paths are interrupted by isotropic scatterings during which the scattered particle sees the direction and the module of its velocity redetermined through a sampling procedure that respects Boltzmann's distribution for the temperature of the medium.

The reactions of the pairs $\left(e^{+}, e^{-}\right)$and $\left(e^{-}, \mathrm{S}^{+}\right)$are assumed to be diffusion controlled. The encounters of the reactive species are considered to occur when their separation becomes smaller than a predefined reaction radius of the order of $0.5 \mathrm{~nm}$. The dielectric constant being close to 2 for the solvents studied here, the (negative) energy of the field dominates the thermal kinetic energy for separations up to a few tens of nanometers and one can show that the actual choice of the reaction radius is not critical. The simulations were achieved up to times of $\sim 500 \mathrm{ps,} \mathrm{after} \mathrm{which} \mathrm{the} \mathrm{prob-}$ ability of Ps formation is negligible. Typically, a thousand runs were necessary to obtain an uncertainty of $1 \%$ on the calculated values of $I_{3}$. 
As a verification of the validity of our simulation code and its algorithm, we studied the motion of electrons in an external electric field $\mathbf{E}$, as well as the geminate recombination of a single $\left(e^{-}, \mathrm{S}^{+}\right)$pair. In the former case, the variation of the simulated drift velocity $\mathbf{v}$ of the electrons with $\mu\left(e^{-}\right)$followed the expected relation $(\mathbf{v}=\mu \mathbf{E}) .{ }^{44}$ In the latter case, we compared the simulated free-ion yields to those predicted by the Onsager theory ${ }^{45}$ for different initial separations and the agreement was always excellent in the range of applicability of the theory. ${ }^{46}$

\section{Results and interpretation}

In order to anchor our sensitivity study to the actual measurements of $I_{3}$, we started from the case of ambient 2,2-DMB, a solvent in which $\mu\left(e^{-}\right) \sim 10 \mathrm{~cm}^{2} \mathrm{~V}^{-1} \mathrm{~s}^{-1}$ is low enough to avoid problems related to the breakdown of the Onsager recombination theory, ${ }^{46}$ and large enough to ensure that electrons are quasifree and undergo very little transient trapping. ${ }^{47}$ Assuming, for simplicity, that the transport properties of $e^{+}$in 2,2-DMB (which are totally unknown) are the same as those of $e^{-}$, we found a good agreement between the simulated $I_{3}$ value and the experimental one $(46 \%)$ at ambient temperature if the mean distance $D$ between successive ionizations in the $e^{+}$track end is $\sim 16.5 \mathrm{~nm}$. The average formation time of Ps was found to be $\sim 18 \mathrm{ps}$, a value which is compatible with the fast rise of the signal in the $\gamma$-emission measurements.

The systematic variation of the parameters of our simulations led to the following main observations.

(i) The multi-ionization nature of the spur is important since only half of the Ps formation could be accounted for by the encounter of $e^{+}$with the last $e^{-}$of the track end. Yet the whole positron track structure does not have to be incorporated in the model since $95 \%$ of the Ps involved one of the last five electrons out of the twenty $e^{-}-\mathrm{S}^{+}$cation pairs considered in our simulations. The values of $I_{3}$ increase with decreasing interionization distance $D$ since it provides more possibilities for the $\left(e^{+}-e^{-}\right)$encounters.

(ii) $I_{3}$ increases with the mean thermalization distance $b$. For example, with $\mu\left(e^{+}\right)=\mu\left(e^{-}\right)=10 \mathrm{~cm}^{2} \mathrm{~V}^{-1} \mathrm{~s}^{-1}, I_{3}$ varies from $43 \%$ to $47 \%$ when the most probable thermalization distance of $e^{-}$and $e^{+}$is brought from 6 to $12 \mathrm{~nm}$. These two $b$ values correspond to those found in the literature for $n$-hexane ${ }^{42}$ and $2,2-\mathrm{DMB},{ }^{41}$ respectively, and are thus typical. The dependence of $I_{3}$ upon $b$ can be attributed to the multi-ionization nature of the studied spur. In fact, an increase in $b$ affects predominantly the $e^{-}+\mathrm{S}^{+}$recombination (as opposed to the $e^{-}+e^{+}$encounter) since the initial separation between the $e^{+}$and the various $e^{-}$also includes the fixed distance $D$ between the successive ionization sites. Simulations performed on single-ionization spurs $(D \rightarrow \infty)$ showed that a change in $b$ affects about equally the two competing processes and does not influence $I_{3}$ significantly.

(iii) $I_{3}$ increases logarithmically with the thermal electron and positron mobilities. Fixing $b$ at a value of $6 \mathrm{~nm}$, we found that $I_{3}$ increases from $35 \%$ to $43 \%$ when both $\mu\left(e^{-}\right)$ and $\mu\left(e^{+}\right)$were brought from 0.1 to $10 \mathrm{~cm}^{2} \mathrm{~V}^{-1} \mathrm{~s}^{-1}$. For the intermediate value of $1 \mathrm{~cm}^{2} \mathrm{~V}^{-1} \mathrm{~s}^{-1}$, the simulated $I_{3}$ was $39 \%$. This variation of $I_{3}$ with the mobility can be in- terpreted in terms of the competition between Ps formation and the first-order decay of the quasifree $e^{+}$. In fact, a change in the mobility does not affect the ultimate probability of recombination of a given pair of charges but modifies its kinetics. At the lowest mobilities considered here, the Ps formation occurs over a few tens of picoseconds and the decay of the $e^{+}$whose characteristic time $\tau_{2}$ is of the order of $450 \mathrm{ps}$ in $n$-hexane (see Fig. 3) is sufficiently competitive to reduce $I_{3}$ by a few percents. This effect is found to be negligible for mobilities above $10 \mathrm{~cm}^{2} \mathrm{~V}^{-1} \mathrm{~s}^{-1}$.

(iv) The role of the temperature as an explicit variable, as opposed to its influence on $\mu$ and $b$ which, in turn, affects $I_{3}$, is mainly to counterbalance the potential energy of the Coulomb field. In this respect, a change in temperature is equivalent to a variation of the dielectric constant of the solvent. A lower temperature therefore leads to a larger recombination probability for a pair of charged reactants. This effect is particularly important for the $\left(e^{-}, \mathrm{S}^{+}\right)$pairs which are less separated than the $\left(e^{-}, e^{+}\right)$pairs. The simulations thus show that $I_{3}$ decreases with decreasing $T$. One must also consider the influence of the temperature on the kinetics of the encounters. In fact, the rate of encounter of the reactants depends on their diffusion coefficients which are related to the product of their mobility with temperature. As was the case when the mobility was reduced (with $T$ kept constant), the sole lowering of $T$ causes a slowing-down of the nonhomogeneous chemistry and, in turn, favors the first-order decay of the $e^{+}$in the liquid. As a consequence of the combination of those two effects, the simulations exhibit a slight reduction (of $\sim 2 \%$ ) of $I_{3}$ when $T$ is lowered from 293 to $213 \mathrm{~K}$.

From this discussion, one can grasp the complexity of the factors that cause the variation of $I_{3}$ which is observed experimentally when the temperature is lowered for a given liquid. The cooling simultaneously changes many relevant physical parameters, such as $b_{e^{-}}, b_{e^{+}}, \mu\left(e^{-}\right), \mu\left(e^{+}\right)$, the dielectric constant, and the decay time $\tau_{2}$. Based on the important assumption that the transport properties of $e^{+}$are similar to those of $e^{-}$, our simulations suggest that, in 2,2DMB and in $n$-hexane, the observed variations of $I_{3}$ with $T$ are mainly caused by its dependence on $\mu\left(e^{-}\right)$and $\mu\left(e^{+}\right)$ that was explained above. The value of $b_{e^{-}}$does not vary by more than $\sim 10 \%$ for a given liquid; it follows approximately the variation of the inverse of the density with $T .^{41}$ As for the variation of $I_{3}$ from one liquid to another at room temperature, it seems to result mainly from a change in the thermalization distances of $e^{-}$and $e^{+}$(for example, $b_{e^{-}}=12.3 \mathrm{~nm}$ in 2,2-DMB and $6.2 \mathrm{~nm}$ in $n$-hexane). ${ }^{42,43}$ The differences in the thermal mobilities of $e^{-}$and $e^{+}$in those two liquids also contribute to a change in $I_{3}$.

One should note that our simulations with low mobilities were meant to show the influence of this parameter on $I_{3}$. They may not provide a satisfying description of Ps formation in $n$-hexane since the $e^{-}$transport in this liquid is known to involve transient trapping ${ }^{35}$ and since very large values of $\mu\left(e^{+}\right)\left(8.5\right.$ and $\left.100 \mathrm{~cm}^{2} \mathrm{~V}^{-1} \mathrm{~s}^{-1}\right)$ can be found in the literature. ${ }^{48,49}$ A preliminary study of the situation using $\mu\left(e^{+}\right)=8.5 \mathrm{~cm}^{2} \mathrm{~V}^{-1} \mathrm{~s}^{-1} \gg \mu\left(e^{-}\right)=0.065 \mathrm{~cm}^{2} \mathrm{~V}^{-1} \mathrm{~s}^{-1}$ is underway and suggests that the measured $I_{3}$ can be repro- 
duced with the simulations if a most probable thermalization distance of $\sim 12.5 \mathrm{~nm}$ is chosen for $e^{+}$, that is, a value which is twice as large as that of $b_{e^{-}}$. This clearly illustrates that the reproduction of the experimental $I_{3}$ results does not determine a unique set of physical properties for the composition of the spur and for the transport properties of the positron.

If we consider higher mobility liquids, such as neopentane and TMS, the simulations show that $I_{3}$ should again increase due to the longer thermalization distances. The explicit influence of $\mu\left(e^{-}\right)$and $\mu\left(e^{+}\right)$were found to be small due to the very short Ps formation time (with which $\tau_{2}$ cannot compete) and to the breakdown of Onsager's recombination theory ${ }^{46}$ that occurs at high mobilities and restrains the probability of an $e^{-}-e^{+}$encounter. Our simulations did not allow us to reproduce the large values of $I_{3}(>50 \%)$ which are observed. Perhaps other factors should be included in the simulations, such as the complex structure of the positron track end or a significant difference between the transport properties of $e^{-}$and those of $e^{+}$.

Finally, the case of Ps formation in polar liquids is worth mentioning. For those solvents, a very fast process competes with the encounter of $e^{+}$and $e^{-}$, namely, the trapping of those two types of particles. We found that the low values of $I_{3}$ observed in those liquids $(\sim 23 \%)$ could be well explained with our model if we take a trapping time of the order of a few hundreds of femtoseconds, in excellent agreement with various experimental determinations of this time. $^{50,51}$ This result supports what was stated in Sec. III A on this issue, that is, the low values of $I_{3}$ in polar solvents are not caused by the low mobilities of the solvated $e^{+}$and $e^{-}$.

\section{CONCLUSIONS}

We have reexamined the proposed correlation between $I_{3}$ and $\mu\left(e^{-}\right)$in liquids along two ways. First, we presented a compilation of $I_{3}$ and $\mu\left(e^{-}\right)$values measured at ambient temperature for 51 different pure liquids. This compilation showed that the (low-mobility) polar liquids constitute a distinct group that displays small values of $I_{3}$. It also showed that, for nonpolar solvents, a positive correlation between $I_{3}$ and $\mu\left(e^{-}\right)$is only apparent among the highest mobility liquids. Second, we measured $I_{3}$ as a function of temperature in three solvents (TMS, 2,2-DMB, and $n$-hexane) covering a wide range of $e^{-}$mobilities. In $n$-hexane and 2,2-DMB, the temperature dependence of $I_{3}$ and $\mu\left(e^{-}\right)$are found to be similar. The fact that this is not the case for TMS may be related to the very large $\mu\left(e^{-}\right)$values in this liquid. It remains unclear, however, why its behavior contrasts with that of neopentane. ${ }^{33}$ Finally, we used Monte Carlo simulation techniques to perform a sensitivity study on the influence of various physical parameters on Ps formation. In the light of those simulations, the problem clearly appears as being multiparametrical. We find, in particular, that the thermalization distances of $e^{+}$and $e^{-}$may constitute the most significant physical parameters influencing Ps formation and that their role relies on the multi-ionization nature of the positron track end. An extension of this kind of work to higher temperatures (up to the critical point) is expected to bring further insight on this question.

\section{ACKNOWLEDGMENTS}

Funding for this research was provided, in part, by the Natural Sciences and Engineering Research Council of Canada and by the Medical Research Council of Canada. This support is herewith gratefully acknowledged.

${ }^{1}$ O. E. Mogensen, J. Chem. Phys. 60, 998 (1974).

${ }^{2}$ J. Ch. Abbé, G. Duplâtre, A. G. Maddock, and A. Haessler, J. Radioanal. Chem. 55, 25 (1980).

${ }^{3}$ J. Ch. Abbé, G. Duplâtre, A. Haessler, and J. Talamoni, Radiat. Phys. Chem. 28, 19 (1986).

${ }^{4}$ A. Haessler, J. Ch. Abbé, and G. Duplâtre, Nucl. Instrum. Methods 174, 317 (1980).

${ }^{5}$ Positron and Positronium Chemistry, edited by D. M. Schrader and Y. C. Jean (Elsevier, Amsterdam, 1988).

${ }^{6}$ A. P. Mills, Jr., J. Chem. Phys 62, 2646 (1975).

${ }^{7}$ I. Billard, J. Ch. Abbé, and G. Duplâtre, Chem. Phys. 127, 273 (1988).

${ }^{8}$ I. Billard, J. Ch. Abbé, and G. Duplâtre, J. Chem. Phys. 97, 1548 (1992).

${ }^{9}$ P. Jansen and O. E. Mogensen, Chem. Phys. 25, 75 (1977).

${ }^{10}$ J.-P. Jay-Gerin, T. Goulet, and I. Billard, Can. J. Chem. 71, 287 (1993), and references therein.

${ }^{11}$ J. Engler, J. Knapp, and G. Vater, Nucl. Instrum. Methods Phys. Res. A 327, 102 (1993).

${ }^{12}$ O. E. Mogensen and F. M. Jacobsen, Chem. Phys. 73, 223 (1982).

${ }^{13}$ Y. Kobayashi, Ber. Bunsenges. Phys. Chem. 96, 1869 (1992).

${ }^{14}$ F. Didierjean, I. Billard, W. F. Magalhães, and G. Duplâtre, Chem. Phys. 174, 331 (1993)

${ }^{15}$ P. R. Gray, C. F. Cook, and G. P. Sturm, Jr., J. Chem. Phys. 48, 1145 (1968)

${ }^{16}$ G. Wikander, N. J. Pedersen, and O. E. Mogensen, Chem. Phys. 86, 213 (1984).

${ }^{17}$ W. F. Magalhães, J. Ch. Abbé, and G. Duplâtre, Chem. Phys. 136, 141 (1989).

${ }^{18}$ B. Lévay, S. J. G. Lund, and O. E. Mogensen, Chem. Phys. 48, 97 (1980).

${ }^{19}$ K. Itoh, M. Nishikawa, and R. A. Holroyd, J. Chem. Phys. 105, 5510 (1996).

${ }^{20}$ O. E. Mogensen, Positron Annihilation in Chemistry (Springer-Verlag, Berlin, 1995), p. 74.

${ }^{21}$ J.-P. Jay-Gerin and C. Ferradini, J. Chim. Phys. 91, 173 (1994), and references therein.

${ }^{22}$ N. Gee and G. R. Freeman, J. Chem. Phys. 90, 5399 (1989).

${ }^{23}$ Z. Zhang, Y. Ito, and Y. Tabata, Radiat. Phys. Chem. 36, 253 (1990).

${ }^{24}$ J.-P. Jay-Gerin and C. Ferradini, in Excess Electrons in Dielectric Media, edited by C. Ferradini and J.-P. Jay-Gerin (CRC Press, Boca Raton, 1991), p. 259; Radiat. Phys. Chem. 38, 83 (1991).

${ }^{25}$ J. A. Riddick, W. B. Bunger, and T. K. Sakano, Organic Solvents. Physical Properties and Methods of Purification, 4th ed. (Wiley, New York, 1986).

${ }^{26}$ I. Billard, J. Ch. Abbé, and G. Duplâtre, Chem. Phys. 184, 365 (1994).

${ }^{27}$ J. Ch. Abbé, G. Duplâtre, A. G. Maddock, and A. Haessler, Radiat. Phys. Chem. 15, 617 (1980).

${ }^{28}$ W. F. Magalhães, J. C. Abbé, and G. Duplâtre, Struct. Chem. 2, 399 (1991).

${ }^{29}$ J. Talamoni, G. Duplâtre, J. Ch. Abbé, and A. Haessler, Chem. Phys. 83, 471 (1984).

${ }^{30}$ C. D. Jonah, J. Ch. Abbé, G. Duplâtre, and A. Haessler, Chem. Phys. 58, 1 (1981)

${ }^{31}$ V. M. Byjakov, V. I. Grafutin, and O. V. Koldaeva, Radiat. Phys. Chem. 10, 239 (1977).

${ }^{32}$ V. L. Bugaenko, V. M. Byakov, V. I. Grafutin, O. V. Koldaeva, and E. V. Minaichev, Radiat. Phys. Chem. 11, 145 (1978).

${ }^{33}$ F. M. Jacobsen, O. E. Mogensen, and G. Trumpy, Chem. Phys. 69, 71 (1982).

${ }^{34} \mathrm{H}$. Stoll and P. Castellaz (private communication).

${ }^{35}$ S. S.-S. Huang and G. R. Freeman, Can. J. Chem. 56, 2388 (1978).

${ }^{36}$ J.-P. Dodelet and G. R. Freeman, Can. J. Chem. 55, 2264 (1977).

${ }^{37}$ I. Billard, T. Goulet, J.-P. Jay-Gerin, and A. Bonnenfant, in Proceedings of the 11th International Conference on Positron Annihilation (ICPA-11), 
Kansas City, Missouri, 1997, edited by Y. C. Jean, M. Eldrup, D. M. Schrader, and R. N. West (Materials Science Forum, Zurich, 1997), p. 99.

${ }^{38}$ N. E. Cipollini and A. O. Allen, J. Chem. Phys. 67, 131 (1977).

${ }^{39}$ D. A. Diehl and D. M. Schrader, J. Chem. Phys. 94, 289 (1991).

${ }^{40}$ T. Azuma, Y. Ito, and Y. Tabata, Radiat. Phys. Chem. 28, 69 (1986).

${ }^{41}$ J.-P. Dodelet, K. Shinsaka, and G. R. Freeman, Can. J. Chem. 54, 744 (1976).

${ }^{42}$ N. Gee, P. C. Senanayake, and G. R. Freeman, J. Chem. Phys. 89, 3710 (1988).

${ }^{43}$ The step-by-step algorithm is thoroughly explained in Ref. 44 for a simple case where the motion of three particles is simulated. One should note that the encounter kinetics cannot be modeled here with the efficient "independent reaction time (IRT)" method since the analytical and numerical formulations of the time-dependent survival probability functions of charged reactants are not accurate enough in low-permittivity media. See, for example, N. J. B. Green, M. J. Pilling, and P. Clifford, Mol. Phys. 67, 1085 (1989).

${ }^{44}$ T. Goulet and J.-P. Jay-Gerin, J. Chem. Phys. 96, 5076 (1992).

${ }^{45}$ L. Onsager, Phys. Rev. 54, 554 (1938).
${ }^{46}$ When $\mu\left(e^{-}\right)$is larger than $\sim 20 \mathrm{~cm}^{2} \mathrm{~V}^{-1} \mathrm{~s}^{-1}$, the electron mean free path ceases to be negligible in comparison to the Onsager radius. This affects the ultimate recombination probability of a geminate electron-cation pair. For a complete description of this "breakdown" of the Onsager theory, see M. Tachiya, J. Chem. Phys. 89, 6929 (1988).

${ }^{47}$ K. Itoh, R. C. Muñoz, and R. A. Holroyd, J. Chem. Phys. 90, 1128 (1989).

${ }^{48}$ F. Heinrich and A. Schiltz, in Proceedings of the 6th International Conference on Positron Annihilation (ICPA-6), Arlington, Texas, 1982, edited by P. G. Coleman, S. C. Sharma, and L. M. Diana (North-Holland, Amsterdam, 1982), p. 705.

${ }^{49}$ S. Linderoth, I. K. MacKenzie, and S. Tanigawa, Phys. Lett. 107A, 409 (1985).

${ }^{50}$ A. Migus, Y. Gauduel, J. L. Martin, and A. Antonetti, Phys. Rev. Lett. 58, 1559 (1987); F. H. Long, H. Lu, and K. B. Eisenthal, Phys. Rev. Lett. 64, 1469 (1990)

${ }^{51}$ M. Sander, U. Brummund, K. Luther, and J. Troe, Ber. Bunsenges. Phys. Chem. 96, 1486 (1992); C. Pépin, T. Goulet, D. Houde, and J.-P. JayGerin, J. Chim. Phys. 93, 182 (1996). 